\title{
Liver Cancer: Zheng Classification of Qi Stagnation and Blood Stasis
}

\author{
Yongsong Guan*, Qing He \\ ${ }^{1}$ Department of Oncology, West China Hospital of Sichuan University, Chengdu, China; ${ }^{2}$ State Key Laboratory of Biotherapy, West \\ China Medical School, Sichuan University, Chengdu, China. \\ Email: *yongsongguan@yahoo.com
}

Received November $29^{\text {th }}, 2013$; revised December $29^{\text {th }}$, 2013; accepted January $7^{\text {th }}, 2014$

Copyright (C) 2014 Yongsong Guan, Qing He. This is an open access article distributed under the Creative Commons Attribution License, which permits unrestricted use, distribution, and reproduction in any medium, provided the original work is properly cited. In accordance of the Creative Commons Attribution License all Copyrights (C) 2014 are reserved for SCIRP and the owner of the intellectual property Yongsong Guan, Qing He. All Copyright @ 2014 are guarded by law and by SCIRP as a guardian.

\section{ABSTRACT}

There is a long history of using traditional Chinese medicine in the treatment of liver cancer and other malignnancies. The classification of Qi stagnation and blood stasis (QSBS) is the most common Zheng in liver cancer. This Zheng is frequently encountered in liver cancers falling into the pathological category of massive type. QSBS is the principal mechanism for a tumor to develop. Liver cancer with QSBS Zheng has characteristic clinical manifestations. Evidence from ex vivo, in vivo and clinical studies has been reported for the identification and management of liver cancer with QSBS Zheng. This article reviews evidence-based study advances of QSBS Zheng, the most common Zheng encountered in liver cancer.

\section{KEYWORDS}

\section{Chinese Traditional Medicine; Syndrome; Liver Cancer}

\section{Introduction}

Liver cancer is one of the leading causes of cancer death worldwide [1]. Several therapeutic modalities have been used for its management [2], but satisfactory results are infrequently obtained [3]. Therefore, development of new treatment strategies is urgently needed for this highly complex disease which is unique in several aspects [4]. Comprehensive, multidisciplinary approaches are searched for improving survival rates of patients with liver cancer [5]. Complementary and alternative medicines are actively sought for this purpose [6], among them, traditional Chinese medicine (TCM) is well known for its long history of application in both treatable and incurable diseases in Asia and the West. Some TCM therapy based on evidence has been reported yielding unexpectedly good results in treatment of liver cancer [7].

Primary liver cancer falls into such disease categories in TCM as "Zhengjia” (mass obstruction in the abdomen), “accumulation”, “distention”, "mass in the liver”, "Fu liang” (amassment), and “jaundice” [8]. TCM practition-

${ }^{*}$ Corresponding author. ers think highly of Zheng Hou in their clinical practices. Zheng denotes evidence and Hou implies clinical manifestations [9]. Identification, differentiation and clarification of a certain Zheng (also known as syndrome) are essential for making a decision of treatment in TCM. A TCM Zheng is in dynamic change caused by multiple correlative factors [10]. It is the highly condensed summing-up of the etiology, nature, location and tendency of a certain disease at a particular stage [11], and indicates the essence of the disease to some extent as the basis of clinical management [12]. According to a modern nomination system, the name of a TCM Zheng (or syndrome pattern) consists of two or more syndrome elements (or factors) which fall into two categories: the nature, and the location of the disease [13]. For example, the Zheng of "heart-liver blood deficiency" is constituted by location elements the heart and the liver, and disease nature element blood deficiency [14]. The main Zheng classifications of liver cancer include blood stasis Zheng, Qi stagnation Zheng, Qi deficiency Zheng, Yin deficiency Zheng, blood deficiency Zheng, Yang deficiency Zheng, excess-heat Zheng, and fluid-dampness Zheng [15]. This 
article reviews evidence-based study advances of QSBS Zheng, the most common Zheng encountered in liver cancer.

\section{TCM Characteristics of Liver Cancer with QSBS Zheng}

Clinical manifestations of liver cancer with QSBS Zheng include painful abdominal mass which is immovable and often soft not firm, wasting and dark face, margin of the tongue looking dark-purple and dotted, thin and white or yellowish tongue-coat [16], unsmooth pulse or taut and thready pulse [17]. These symptoms and signs are frequently correlated to QSBS Zheng. For example, constipation, a common problem in advanced cancer patients, can be an excessive Zheng and characterized by pathological accumulation of qi. Thus constipation is often classified as with qi stagnation Zheng. One study analyzed data of 225 advanced cancer patients with constipation and demonstrated that $40.0 \%$ of them (90 patients) were classified as with qi stagnation Zheng manifested by one or more dominant symptoms and signs such as dry mouth, tiredness, agitation, belching, anorexia, pale red tongue, and fine or string-like pulse [18].

QSBS is both the process and the consequence of a tumor to develop which explains that this Zheng is the mostly encountered in liver cancer [15]. When liver cancer presents as a single Zheng, it manifests most frequently as qi stagnation Zheng, and then, blood stasis Zheng. QSBS Zheng is the concurrent existence of both qi stagnation and blood stasis Zhengs. A recent study identified distribution features of single Zheng in 106 patients with liver cancer before treatment and found that blood stasis (86.8\%) and qi stagnation (56.5\%) were the first and the second commonest Zhengs, respectively [15]. Asthenic healthy energy and sthenic evil is the pathogenesis of cancer development. The interaction or fight between healthy energy and evil runs through the entire process of a cancerous disease. When external evil goes strong, the internal healthy energy could be weakened. A recent experimental study demonstrated the establishment of QSBS rat model by vigorous external evil. When combination use of vicious stimulation including noise, light, electricity, tail-clamping, bondage and icewater-bath was applied to, all the model animals showed significant reduction in the blood flow velocity in mesenteric microcirculation and increase in the whole blood viscosity, the plasma viscosity and the fibrinogen content [19].

Chronic hepatitis B is believed to be one of the main causes for liver cancer. Recently, some investigators analysed plasma proteome from cases of different TCM Zhengs in patients with chronic hepatitis B. The results showed that in cases of liver-qi stagnation and spleen deficiency Zheng, and blood stasis into collaterals Zheng, level of immunoglobulin J-chains is up-regulated and significantly related to the certain Zheng, implying that level of immunoglobulin J-chains could be used as a novel potential biomarker for diagnosis of a certain Zheng in patients with chronic hepatitis B [20]. In a study investigating 793 patients with fatty liver disease which is another cause of liver cancer, liver-qi stagnation Zheng was determined as one of the pathogenic factors [21]. Another study showed that in patients having chronic hepatitis B, 75.0\% of the cases with QSXS Zheng were in the early stage of cirrhosis which is significantly associated with liver cancer [22].

TCM practitioners often have controversial opinions about Zheng classifications and the connotation even in the same Zheng is decided based on their own clinical experiences, which makes it difficult to draw a consensual conclusion for diagnosis and decision for treatment [23]. Nevertheless, TCM legacy in general from several millennia and its modernized version are well-known worldwide for their healing effects. In addition, highquality efficacy in its management of chronic diseases has proved the success in treating disease such as hepatolenticular degeneration by integrated TCM and Western medical therapies with report success rates as high as 85\% [24]. Thus a thoughtfully developed standardized approach is needed to include its phenomenological, philosophical, and methodological aspects for the comprehension of the unique characteristics of TCM treatment [25]. Several diagnostic criteria for basic Zhengs of TCM have been published, including QSXS Zheng which is the most frequent in patients with primary liver cancer $[11,13,26]$. These criteria are developed by the collection, analysis and generalization of related medical literature, clinical investigation, and experts' opinions. However, before they become satisfactory and practical, repeated clinical verification, revision and modification are warranted.

Tongue inspection is an important, non-invasive, simple, unique and valuable diagnostic method in TCM. Many signs are used in the discrimination of Zhengs, but the tongue-coating appearance is one of the major elements [27]. One clinical control study observed the distribution of 10 abnormal tongue signs in 104 patients with 7 different Zhengs. Patients in the QSBS Zheng group had all the 10 abnormal tongue signs [28]. The sign of dark blue and purple tongue presents more frequently in liver cancer patients with QSBS Zheng [16, 29].

QSXS Zheng is frequently encountered in liver cancers falling into the pathological category of massive type. According to a clinical research of 203 patients, the commonest manifestation in patients with liver cancer of qi stagnation Zheng is abdominal distention, while that of 
blood stasis Zheng is mass in the right upper quadrant [30]. Table 1 shows the main TCM characteristics of liver cancer with QSBS Zheng.

\section{Differentiation from Liver Cancer with Other Zheng Classifications}

The third single Zheng by frequency of occurrence presented in liver cancer is spleen-qi deficiency Zheng, and the following in turn are liver-yin deficiency, kidney-yin deficiency, hepatochlic hygropyrexia, and evil toxin predominance Zhengs [8]. As liver cancer is one of the most common types of cancer, it is necessary to differentiate these Zhengs from QSBS Zheng.

Patients with spleen-qi deficiency often have decreased immunity, manifested by vague pain below xyphoid which can be relieved by pressing and warming [31], anorexia, nausea, vomiting, diarrhea, and pale faces and lips [32]. Such symptoms as tiredness, fatigue, loose stool, and poor appetite are significantly correlated to cancer [33].

Patients with liver-yin deficiency can have flank pain, dryness of eyes, dizziness and tinnitus, burning sensation of five centers, feverish dysphoria, dry pharynx, wriggling of limbs, amenorrhea or scanty menstruation, stringy, thin and rapid pulse, red tongue with sparse Saliva and coat $[33,34]$.

Symptoms of kidney-yin deficiency Zheng fall into the category of "heat". These include sore waist and knees, skelasthenia, vertigo and tinnitus, insomnia and dreaminess, postcoital protrusion or impotence or nocturnal emission in man, hypomenorrhea or menostasis even uterine bleeding in woman, emaciation, feverish night sweating, burning sensation of five centers, dry pharynx and red cheeks, juvenile leukotrichia, somniloquence and bruxism, frequency of micturition, dark yellow urine, dry stool, red tongue with sparse saliva, rapid and thready pulse $[33,35,36]$.

Symptoms of hepatochlic hygropyrexia Zheng include hypochondrium distending pain, bitter taste of mouth and anorexia, nausea and vomiting, abdominal distention, discordant stool, scanty dark urine, jaundice, alternate chill and fever, eczema of scrotum, swelling testes, thermalgia, yellow and foul morbid leucorrhea, pruritus of vulva, red tongue with yellowish and greasy fur, and stringy, smooth and rapid pulse $[17,33,35]$. This Zheng can be confused with splenogastric hygropyrexia Zheng and should be differentiated carefully [37].

Evil toxin predominance Zheng is featured by acute onset of disease, feverish restlessness, yellowish skin, faces and sclera, hepatosplenomegaly, even obnubilation, convulsion, sweating, dark yellow urine, obstipation, foul morbid leucorrhea, purple-red tongue with yellow thick fur, rapid and floating pulse [17,33,38].

In addition, attention should be paid to the dynamic change of Zhengs, and to the situation that different Zhengs exist in the same disease and the same Zheng exists in different diseases. Table 2 presents the differences to discriminate QSBS Zheng from other Zheng classifications of liver cancer.

\section{TCM Management of Liver Cancer with QSBS Zheng}

The essence of a cancerous process is that pathogen being excessive but vital qi weak. In the treatment of cancer, TCM plays its role in driving away evil pathogen and supporting body resistance, i.e., vital qi. Currently, Western oncology has an integrated model to function. The conventional methods are taken as the mainstream, and TCM is accepted as one complementary and alternative method, including its therapeutic interventions [39]. Synergistic therapeutic effects can be attained by integrating conventional methods and TCM. For treatment of liver cancer, several mainstream modalities are practiced at the present, including surgery, local ablation, and chemoembolization $[40,41]$. Transcatheter arterial chemoembolization (TACE) is the most widely used mainstream method for liver cancer and is considered the gold standard for treating intermediate-stage hepatocellular carcinoma because of its survival benefit which can be improved by the combining use of TCM method [42,43]. We have participated in a study through a meta-analysis of 37 randomized controlled trials involving 2653 liver cancer patients to compare the efficacy and safety of TACE integrating TCM with that of TACE alone. The results showed that TACE integrating TCM improved patient survival, quality of life, alleviation of symptoms, and tumor response, and was thus more the- rapeutically beneficial than TACE alone [44]. Another systematic review and meta-analysis included 45 randomized controlled trials involving 3236 liver cancer patients found compelling evidence of a role for TCM in liver cancer [45]. Although consistent effects of TCM were reported

Table 1. Characteristics of liver cancer with QSBS Zheng.

\begin{tabular}{ll}
\hline \multicolumn{1}{c}{ Description } & Characteristics \\
\hline Palpable abdominal mass & Painful, immovable, soft \\
TCM inspection & Wasting and dark face, dark-purple and dotted margin of tongue, thin and white or yellowish tongue-coat \\
Pulse-feeling & Unsmooth or taut and thready \\
\hline
\end{tabular}


Table 2. Differentiation from other Zheng classifications of liver cancer.

\begin{tabular}{|c|c|}
\hline Zheng classification & Characteristics for differentiation \\
\hline Qi stagnation Zheng & Abdominal distention, eructation, mobile gas pains, string pulse, normal tongue sign \\
\hline Blood stasis Zheng & $\begin{array}{l}\text { Immovable mass in the right upper quadrant, fixed unpressable sharp pain, melena, dark complexion, } \\
\text { scaly skin, violet oral lips and onyx, subcutaneous peliona, spider nevi, varicosity of abdominal wall, } \\
\text { menostasis, dark purple tongue, unsmooth thin pulse }\end{array}$ \\
\hline Spleen-qi deficiency Zheng & Vague pain below xyphoid, tiredness, fatigue, loose stool, and poor appetite \\
\hline Liver-yin deficiency & $\begin{array}{l}\text { Flank pain, dryness of eyes, dizziness and tinnitus, burning sensation of five centers, feverish dysphoria, } \\
\text { dry pharynx, wriggling of limbs, amenorrhea or scanty menstruation, stringy, thin and rapid pulse, } \\
\text { red tongue with sparse saliva and coat }\end{array}$ \\
\hline Kidney-yin deficiency & $\begin{array}{l}\text { Sore waist and knees, skelasthenia, vertigo and tinnitus, insomnia and dreaminess, postcoital protrusion } \\
\text { or impotence or nocturnal emission in man, hypomenorrhea or menostasis even uterine bleeding in woman, } \\
\text { emaciation, feverish night sweating, burning sensation of five centers, dry pharynx and red cheeks, juvenile } \\
\text { leukotrichia, somniloquence and bruxism, frequency of micturition, dark yellow urine, dry stool, red tongue } \\
\text { with sparse saliva, rapid and thready pulse }\end{array}$ \\
\hline $\begin{array}{l}\text { Hepatochlic hygropyrexia } \\
\text { Zheng }\end{array}$ & $\begin{array}{l}\text { Hypochondrium distending pain, bitter taste of mouth and anorexia, nausea and vomiting, abdominal distention, } \\
\text { discordant stool, scanty dark urine, jaundice, alternate chill and fever, eczema of scrotum, swelling testes, } \\
\text { thermalgia, yellow and foul morbid leucorrhea, pruritus of vulva, red tongue with yellowish and greasy fur, and } \\
\text { stringy, smooth and rapid pulse }\end{array}$ \\
\hline $\begin{array}{l}\text { Evil toxin predominance } \\
\text { Zheng }\end{array}$ & $\begin{array}{l}\text { Acute onset of disease, feverish restlessness, yellowish skin, faces and sclera, hepatosplenomegaly, even } \\
\text { obnubilation, convulsion, sweating, dark yellow urine, obstipation, foul morbid leucorrhea, purple-red } \\
\text { tongue with yellow thick fur, rapid and floating pulse }\end{array}$ \\
\hline
\end{tabular}

when combined with TACE versus TACE alone, publiccation bias and low quality of trial may exist, favoring positive reports. Hence, further large-scale well-conducted trials are warranted and should be conducted in Western settings of double blind, placebo controlled trials.

The correlation between qi and blood can be summarized as that "qi commands blood, while blood fertilizes qi”. Based on the main Zheng classifications of liver cancer as described above, we should pay attention to regulating qi, replenishing qi, removing blood stasis, and clearing heat for treating liver cancer patients $[19,28]$. A clinical study of 106 patients with liver cancer revealed that before TACE blood stasis Zheng and qi stagnation Zheng were predominant. However, after TACE blood stasis Zheng and qi deficiency Zheng became predominant, while the percentage of qi stagnation Zheng decreased and that of excess-heat Zheng, qi deficiency Zheng, blood deficiency Zheng, and yang deficiency Zheng increased [15]. The results of this study suggest that we should mind blood stasis Zheng and removing stasis both before and after TACE. And after TACE, other Zhengs form QSXS Zheng should be paid attention to, by actual observation of the dynamic change of Zhengs.

The method of promoting blood circulation and removing blood stasis has been proposed as a therapeutic approach for patients with liver cancer, especially those with QSBS Zheng. Blood stasis can promote tumor growth and angiogenesis which can be antogonized with TCM treatment in vitro and in vivo [46]. Ex vivo study indicated that TCM treatment by promoting blood circulation and removing blood stasis down-regulates correlated cancer genes in rats with liver cancer [47]. In clinical practice, several decoctions with this function have proved to improve clinical symptoms, signs and quality of life of patients with liver cancer $[8,15]$.

However, some studies have shown evidence that the method of promoting blood circulation and removing blood stasis contributes to cancer metastasis and blood stasis Zheng may inhibit cancer growth and metastasis. This issue was supported by the fact that animals in the status of blood stasis have their tumors growing slowly and less liver metastasis [48]. So the method of promoting blood circulation and removing blood stasis must be used with caution and multiple factors should be considered.

Two decoctions Ge-Xia-Zhu-Yu-Tang and Xue-FuZhu-Yu-Tang, which were proposed in a book called Correction on Errors of Medical Works from the Chin Dynasty, have long been used to treat liver cancer with QSBS Zheng [49,50]. Antitumor activities were indicated when these decoctions were applied to animals bearing experimental liver tumors, showing markedly increased the mean survival time and toxicity to tumor cells and inhibition of synthesis rates of DNA, RNA, and protein. A recent in vivo study demonstrated that Ge-XiaZhu-Yu-Tang also have both hepatoprotective and antifibrogenic effects and may be useful in preventing the development of hepatic fibrosis [51].

Another decoction San-Huang-Xie-Xin-Tang has also been widely used for liver disease including liver fibrosis 
and cirrhosis, with hepatoprotective potency by its anti-inflammation and anti-toxicity effects, and with antitumor potency by its anti-proliferative effect [52]. Evidence of ex vivo study showed that this decoction can activate the most common antitumor gene p53 in liver cancer cell Hep G2 [53].

Evidence should be gained of the effects for each of the mostly used herbs, couple herbs, group of herbs, and formula in prescriptions treating liver cancer with QSBS Zheng [54,55]. Herbs which are most frequently used are those for replenishing qi and resolving stagnation, activating blood circulation to remove blood stasis, removing toxic materials to inhibit tumor growth, and invigorating the spleen to promote appetite. These herbs are mostly of neutral or cool nature, and mainly of sweet, bitter, or pungent taste [8,56]. For example, the herb pair of peach seed and Radix Paeoniae Rubra is commonly used for blood stasis Zheng [54,57], while the group of Curcuma wenyujin, Akebia trifoliata and Bupleurum chinense is potent for qi stagnation Zheng [54,58].

Cupping-bloodletting therapy of Saudi Arabia is also indicated to the treatment of QSBS Zheng by professional practitioners of acupuncture and moxibustion [59]. Harmonious flow of qi is the basis of good health. Acupuncture focuses on restoring qi by manipulation of the complementary and opposing elements of yin and yang into harmony, the ultimate aim in TCM concept of wellbeing [60,61]. Evidence for efficacy in liver cancer with QSBS Zheng can likewise be excavated in ethnomedicines, and other traditional medicine such as Ayurveda and Unani medicine for their characteristic herbal preparations [62,63].

In TCM ideology, physical and mental health is assumed to be determined by the ever-changing but harmonious balance between external and internal forces. Following the prognosis of a disease, TCM practitioners pay special attention to experiences which are superior in treatments before sick described in Huang Di Nei Jing (Inner Canon). This is somewhat like chemoprevention, because we can identify QSXS Zheng at the stage of hepatitis virus infection or cirrhosis. Therefore, the management of liver cancer with prescriptions for QSXS Zheng should start as early as treating patients who suffer hepatitis virus infection or cirrhosis and are classified as with QSXS Zheng.

\section{Conclusion}

It is acknowledged that many of TCM treatments have enormous potential and could have more extensive uses, including those for liver cancer with QSXS Zheng. QSBS is the principal mechanism for a tumor to develop, and is both the process and consequence of a tumor in growth. Evidence supports this view with the phenomenon that coagulation factors and fibrinogen increase in most patients with cancer. QSBS is the most common Zheng in liver cancer. Blood stasis could be a protective tool of the host to confine the tumor and inhibit metastasis. So the method of promoting blood circulation and removing stasis must be used with discretion. Current single herbs, herb pairs, groups and combinations need to be examined one after another for the treatment of liver cancer with QSBS Zheng. The authors have to continue searching for single herbs, herb pairs, groups and combinations for the treatment of liver cancer with QSBS Zheng. Further large-scale well-conducted trials are warranted and should be conducted in western settings of double blind, placebo controlled trials to establish the most appropriate prescriptions for liver cancer with QSBS Zheng.

\section{REFERENCES}

[1] J. F. Bridges, L. Dong, G. Gallego, B. M. Blauvelt, S. M. Joy and T. M. Pawlik, "Prioritizing Strategies for Comprehensive Liver Cancer Control in Asia: A Conjoint Analysis,” BMC Health Services Research, Vol. 12, No. 1, 2012, p. 376.

http://dx.doi.org/10.1186/1472-6963-12-376

[2] T. Livraghi, H. Mäkisalo and P. D. Line, "Treatment Options in Hepatocellular Carcinoma Today," Scandinavian Journal of Surgery, Vol. 100, No. 1, 2011, pp. 22-29.

[3] A. C. Chan, R. T. Poon, T. T. Cheung, et al., "Survival Analysis of Re-Resection versus Radiofrequency Ablation for Intrahepatic Recurrence after Hepatectomy for Hepatocellular Carcinoma," World Journal of Surgery, Vol. 36, No. 1, 2012, pp. 151-156.

http://dx.doi.org/10.1007/s00268-011-1323-0

[4] Q. Gao, Y. Shi, X. Wang, J. Zhou, S. Qiu and J. Fan, "Translational Medicine in Hepatocellular Carcinoma," Frontiers of Medicine, Vol. 6, No. 2, 2012, pp. 122-133. http://dx.doi.org/10.1007/s11684-012-0193-7

[5] R. Wong and C. Frenette, "Updates in the Management of Hepatocellular Carcinoma," Gastroenterology and Hepatology, Vol. 7, No. 1, 2011, pp. 16-24.

[6] J. M. Luk, X. Wang, P. Liu, et al., "Traditional Chinese Herbal Medicines for Treatment of Liver Fibrosis and Cancer: From Laboratory Discovery to Clinical Evaluation,” Liver International, Vol. 27, No. 7, 2007, pp. 879890. http://dx.doi.org/10.1111/j.1478-3231.2007.01527.x

[7] Z. Sun, S. T. Liang, X. F. Zhai, et al., "A Traditional Chinese Herbal Medicine Compound Preparation versus Interventional Therapy after Resection of Small Hepatocellular Carcinoma: 22-Year Follow-Up,” Journal of Traditional Chinese Medicine, Vol. 32, No. 2, 2012, pp. 156-163.

http://dx.doi.org/10.1016/S0254-6272(13)60005-9

[8] X. Liu and N. Li, "Regularity Analysis on Clinical Treatment in Primary Liver Cancer by Traditional Chinese Medicine," China Journal of Chinese Materia Medica, Vol. 37, No. 9, 2012, pp. 1327-1331. 
[9] Z. Y. Xu, "Evidence-Based Medicine and Treatment of Lung Cancer," Journal of Chinese Integrative Medicine, Vol. 1, No. 2, 2003, pp. 151-154. http://dx.doi.org/10.3736/jcim20030221

[10] Q. H. Fu, J. Pei, J. R. Hui and Y. Song, "Studies on Dynamic Changes in Traditional Chinese Medicine Syndrome Patterns for Stroke Using Data-Driven and Model-Driven Approaches: A Review," Journal of Chinese Integrative Medicine, Vol. 9, No. 12, 2011, pp. 12921300. http://dx.doi.org/10.3736/jcim20111203

[11] K. Qu, T. F. Wang, Y. Zhao, et al., "Common Syndrome Factors of Traditional Chinese Medicine in Chronic Renal Failure Based on the Questionnaire Investigation among Experts," Journal of Chinese Integrative Medicine, Vol. 8, No. 12, 2010, pp. 1147-1152. http://dx.doi.org/10.3736/jcim20101206

[12] C. Chen, Y. M. Meng, P. Zhang, et al., "Diagnosis and Treatment Rule of Traditional Chinese Medicine for Syndrome Factors of Chronic Congestive Heart Failure: A Study Based on Shannon Entropy Method,” Journal of Chinese Integrative Medicine, Vol. 8, No. 11, 2010, pp. 1080-1084. http://dx.doi.org/10.3736/jcim20101113

[13] T. F. Wang, C. F. Du, Q. G. Wang, et al., "A Perspective of Developing Syndrome Diagnostic Criteria Based on Syndrome Factors and Combination of Disease in Modern Medicine System and Syndrome in Traditional Chinese Medicine System," Journal of Chinese Integrative Medicine, Vol. 7, No. 10, 2009, pp. 901-906. http://dx.doi.org/10.3736/jcim20091001

[14] T. F. Wang, J. J. Wang, X. L. Xue, et al., "Distribution Characteristics of Traditional Chinese Medicine Syndromes and Their Elements in People with Subhealth Fatigue," Journal of Chinese Integrative Medicine, Vol. 8, No. 3, 2010, pp. 220-223. http://dx.doi.org/10.3736/jcim20100304

[15] Y. H. Zhang, X. Qin and J. Xu, "Analysis of Chinese Medical Syndrome Features of Patients with Primary Liver Cancer before and after Transcatheter Arterial Chemoembolization," Chinese Journal of Integrated Traditional and Western Medicine, Vol. 32, No. 9, 2012, pp. 11711174.

[16] Q. Liu, X. Q. Yue, W. Z. Deng and R. Z. Ren, "Quantitative Study on Tongue Color in Primary Liver Cancer Patients by Analysis System for Comprehensive Information of Tongue Diagnosis," Journal of Chinese Integrative Medicine, Vol. 1, No. 3, 2003, pp. 180-183. http://dx.doi.org/10.3736/jcim20030310

[17] D. Z. Sun, L. Liu, J. P. Jiao, P. K. Wei, L. D. Jiang and L. $\mathrm{Xu}$, "Syndrome Characteristics of Traditional Chinese Medicine: Summary of a Clinical Survey in 767 Patients with Gastric Cancer," Journal of Chinese Integrative Medicine, Vol. 8, No. 4, 2010, pp. 332-340. http://dx.doi.org/10.3736/jcim20100406

[18] C. W. Cheng, A. O. Kwok, Z. X. Bian and D. M. Tse, "The Quintessence of Traditional Chinese Medicine: Syndrome and Its Distribution among Advanced Cancer Patients with Constipation,” Evidence-Based Complementary and Alternative Medicine, Vol. 2012, 2012, Article ID: 739642. http://dx.doi.org/10.1155/2012/739642
[19] T. Wang, C. Jia, Y. Chen, X. Li and J. Cheng, “Analysis on Establishment and Affecting Factors of Qi Stagnation and Blood Stasis Rat Model,” China Journal of Chinese Materia Medica, Vol. 37, No. 11, 2012, pp. 1629-1633.

[20] Y. Liu, P. Liu, R. Dai, et al., “Analysis of Plasma Proteome from Cases of the Different Traditional Chinese Medicine Syndromes in Patients with Chronic Hepatitis B,” Journal of Pharmaceutical and Biomedical Analysis, Vol. 59, 2012, pp. 173-178. http://dx.doi.org/10.1016/j.jpba.2011.10.002

[21] H. F. Wei, T. Liu, L. J. Xing, P. Y. Zheng and G. Ji, "Distribution Pattern of Traditional Chinese Medicine Syndromes in 793 Patients with Fatty Liver Disease," Journal of Chinese Integrative Medicine, Vol. 7, No. 5, 2009, pp. 411-417. http://dx.doi.org/10.3736/jcim20090503

[22] K. Z. Liu, "Relation between Traditional Chinese Medicine Differentiation of Syndromes and HBV Antigen, HBV DNA in Serum and Liver Tissues and Pathological Changes in Chronic Hepatitis B," Chinese Journal of Integrated Traditional and Western Medicine, Vol. 12, No. 1, 1992, pp. 11-13.

[23] H. Uzuner, R. Bauer, T. P. Fan, et al., "Traditional Chinese Medicine Research in the Post-Genomic Era: Good Practice, Priorities, Challenges and Opportunities,” Journal of Ethnopharmacology, Vol. 140, No. 3, 2012, pp. 458-468. http://dx.doi.org/10.1016/j.jep.2012.02.028

[24] X. P. Wang, W. F. Zhang, H. Y. Huang and M. Preter, "Neurology in the People's Republic of China-An Update,” European Neurology, Vol. 64, No. 6, 2010, pp. 320-324. http://dx.doi.org/10.1159/000321648

[25] S. Xutian, D. Cao, J. Wozniak, J. Junion and J. Boisvert, "Comprehension of the Unique Characteristics of Traditional Chinese Medicine,” The American Journal of Chinese Medicine, Vol. 40, No. 2, 2012, pp. 231-244. http://dx.doi.org/10.1142/S0192415X12500188

[26] C. Q. Ling, Q. Liu, D. T. Li, et al., "Study of a Qualitative Diagnostic Criterion for Basic Syndromes of Traditional Chinese Medicine in Patients with Primary Liver Cancer,” Journal of Chinese Integrative Medicine, Vol. 3, No. 2, 2005, pp. 95-98. http://dx.doi.org/10.3736/jcim20050204

[27] B. Jiang, X. Liang, Y. Chen, et al., "Integrating NextGeneration Sequencing and Traditional Tongue Diagnosis to Determine Tonguecoating Microbiome," Scientific Reports, Vol. 2, No. 936, 2012.

[28] J. H. Qin and B. Liu, "Tongue Tip Microcirculation in Patients with Different Symptoms," Chinese Journal of Integrated Traditional and Western Medicine, Vol. 10, No. 10, 1990, pp. 606-608.

[29] T. S. Chen and P. S. Chen, "The Liver in Traditional Chinese Medicine," Journal of Gastroenterology and Hepatology, Vol. 13, No. 4, 1998, pp. 437-442. http://dx.doi.org/10.1111/j.1440-1746.1998.tb00662.x

[30] D. T. Li, C. Q. Ling, Q. B. Lang ,et al., "Construction of a Therapeutic Effect Evaluation System for Patients with Primary Liver Cancer Based on Syndrome Differentiation in Traditional Chinese Medicine," Journal of Chinese Integrative Medicine, Vol. 5, No. 1, 2007, pp. 15-22. 
http://dx.doi.org/10.3736/jcim20070104

[31] G. Xiong, C. Virasakdi, A. Geater, Y. Zhang, M. Li and S. Lerkiatbundit, "Factor Analysis on Symptoms and Signs of Chronic Low-Back Pain Based on Traditional Chinese Medicine Theory," Journal of Alternative and Complementary Medicine, Vol. 17, No. 1, 2011, pp. 51-55.

[32] Q. Zhang, P. Liu, H. F. Cheng, et al., "Clinical Investigation on Characteristics of Traditional Chinese Medical Syndrome of Hepatocirrhosis," Journal of Chinese Integrative Medicine, Vol. 1, No. 2, 2003, pp. 108-112. http://dx.doi.org/10.3736/jcim20030207

[33] F. G. Hou, Y. Cen, J. Guan, L. Y. Zhu and X. L. Yin, "Quantified Diagnositic Standard for Large Intestinal Cancer of Spleen Qi Deficiency Syndrome,” Journal of Chinese Integrative Medicine, Vol. 7, No. 9, 2009, pp. 814-818. http://dx.doi.org/10.3736/jcim20090903

[34] Y. Feng, Z. Wu, X. Zhou, Z. Zhou and W. Fan, "Knowledge Discovery in Traditional Chinese Medicine: State of the Art and Perspectives," Artificial Intelligence in Medicine, Vol. 38, No. 3, 2006, pp. 219-236. http://dx.doi.org/10.1016/j.artmed.2006.07.005

[35] R. Q. Chen, C. M. Wong, K. J. Cao and T. H. Lam, “An Evidence-Based Validation of Traditional Chinese Medicine Syndromes," Complementary Therapies in Medicine, Vol. 18, No. 5, 2010, pp. 199-205. http://dx.doi.org/10.1016/j.ctim.2010.05.036

[36] R. Q. Chen, C. M. Wong and T. H. Lam, "Construction of a Traditional Chinese Medicine Syndrome-Specific Outcome Measure: The Kidney Deficiency Syndrome Questionnaire (KDSQ)," BMC Complementary and Alternative Medicine, Vol. 6, No. 12, 2012, p. 73. http://dx.doi.org/10.1186/1472-6882-12-73

[37] L. Hu, S. X. Lao, Z. Y. Kuang and M. Cheng, “Thoughts on Pathogenesis of Helicobacter Pylori Related Gastrosis with Splenogastric Hygropyrexia Syndrome,” Journal of Chinese Integrative Medicine, Vol. 6, No. 6, 2008, pp. 565-568. http://dx.doi.org/10.3736/jcim20080604

[38] W. Liu, X. Y. Liu and H. B. Zhang, "Exploration into Relationship between TCM Syndrome Type and Laboratory Indexes in Patients with Systemic Lupus Erythematosus," Chinese Journal of Integrated Traditional and Western Medicine, Vol. 28, No. 2, 2008, pp. 115-117.

[39] G. J. Dobos, B. Kirschbaum and K. E. Choi, "The Western Model of Integrative Oncology: The Contribution of Chinese Medicine," Chinese Journal of Integrative Medicine, Vol. 18, No. 9, 2012, pp. 643-651. http://dx.doi.org/10.1007/s11655-012-1200-1

[40] J. J. Lin, C. N. Jin, M. L. Zheng, X. N. Ouyang, J. X. Zeng and X. H. Dai, "Clinical Study on Treatment of Primary Hepatocellular Carcinoma by Shenqi Mixture Combined with Microwave Coagulation," Chinese Journal of Integrative Medicine, Vol. 11, No. 2, 2005, pp. 104-110. http://dx.doi.org/10.1007/BF02836465

[41] P. Song, R. G. Tobe, Y. Inagaki, N. Kokudo, K. Hasegawa, Y. Sugawara and W. Tang, "The Management of Hepatocellular Carcinoma around the World: A Comparison of Guidelines from 2001 to 2011," Liver International, Vol. 32, No. 7, 2012, pp. 1053-1063. http://dx.doi.org/10.1111/j.1478-3231.2012.02792.x
[42] Y. Yu, Q. Lang, Z. Chen, B. Li, C. Q. Yu, D. Z. Zhu, X. F. Zhai and C. Q. Ling, "The Efficacy for Unresectable Hepatocellular Carcinoma May Be Improved by Transcatheter Arterial Chemoembolization in Combination with a Traditional Chinese Herbal Medicine Formula: A Retrospective Study,” Cancer, Vol. 115, No. 22, 2009, pp. 5132-5138. http://dx.doi.org/10.1002/cncr.24567

[43] J. L. Raoul, B. Sangro, A. Forner, V. Mazzaferro, F. Piscaglia, L. Bolondi and R. Lencioni, "Evolving Strategies for the Management of Intermediate-Stage Hepatocellular Carcinoma: Available Evidence and Expert Opinion on the Use of Transarterial Chemoembolization," Cancer Treatment Reviews, Vol. 37, No. 3, 2011, pp. 212-220. http://dx.doi.org/10.1016/j.ctrv.2010.07.006

[44] M. B. Meng, Y. L. Cui, Y. S. Guan, Z. Ying, M. H. Zheng, C. K. Yuan and R. M. Zhang, "Traditional Chinese Medicine plus Transcatheter Arterial Chemoembolization for Unresectable Hepatocellular Carcinoma,” Journal of Alternative and Complementary Medicine, Vol. 14, No. 8, 2008, pp. 1027-1042.

http://dx.doi.org/10.1089/acm.2008.0060

[45] P. Wu, J. J. Dugoua, O. Eyawo and E. J. Mills, "Traditional Chinese Medicines in the Treatment of Hepatocellular Cancers: A Systematic Review and Meta-Analysis," Journal of Experimental \& Clinical Cancer Research, Vol. 28, 2009, p. 112.

http://dx.doi.org/10.1186/1756-9966-28-112

[46] J. X. Yang, I. Fichtner, M. Becker, M. Lemm and X. M. Wang, "Anti-Proliferative Efficacy of Icariin on HepG2 Hepatoma and Its Possible Mechanism of Action," American Journal of Chinese Medicine, Vol. 37, No. 6, 2009, pp. 1153-1165. http://dx.doi.org/10.1142/S0192415X09007569

[47] D. Guan, Z. Fang, H. Lu and H. Li, "Preliminary Investigation on Regulating Effects of Different TCM Treatments on Transcription of the Correlated Genes of Liver Cancer in Rats,” Journal of Traditional Chinese Medicine, Vol. 23, No. 1, 2003, pp. 62-66.

[48] Z. Chen, L. M. Liu and Y. B. He, "Correlation between TXB2, 6-Keto-PGF1 $\alpha$ and Liver Metastasis in Rats Model with Blood Stasis," Journal of Chinese Integrative Medicine, Vol. 1, No. 3, 2003, pp. 199-201. http://dx.doi.org/10.3736/jcim20030316

[49] J. S. You, H. F. Huang and D. M. Hau, "Effects of Xuefu Zhuyu Tang and Mitomycin C on Liver Tumors in Mice," Chang Gung Medical Journal, Vol. 26, No. 6, 2003, pp. 417-424.

[50] T. Chen, D. Li, Y. L. Fu and W. Hu, "Screening of QHF Formula for Effective Ingredients from Chinese Herbs and Its Anti-Hepatic Cell Cancer Effect in Combination with Chemotherapy," Chinese Medical Journal, Vol. 121, No. 4, 2008, pp. 363-368.

[51] J. Y. Chen, H. L. Chen, J. C. Cheng, H. J. Lin, Y. T. Tung, C. F. Lin and C. M. Chen, "A Chinese Herbal Medicine, Gexia-Zhuyu Tang (GZT), Prevents DimethylnitrosamineInduced Liver Fibrosis through Inhibition of Hepatic Stellate Cells Proliferation,” Journal of Ethnopharmacology, Vol. 142, No. 3, 2012, pp. 811-818. http://dx.doi.org/10.1016/j.jep.2012.06.005 
[52] S. Huang, S. J. Chang, M. Yang, J. J. Chen and W. H. Chang, "Nanoscale Hepatoprotective Herbal Decoction Attenuates Hepatic Stellate Cell Activity and ChloroformInduced Liver Damage in Mice,” International Journal of Nanomedicine, Vol. 6, 2011, pp. 1365-1371. http://dx.doi.org/10.2147/IJN.S19503

[53] W. Y. Cheng, S. L. Wu, C. Y. Hsiang, et al., "Relationship Between San-Huang-Xie-Xin-Tang and Its Herbal Components on the Gene Expression Profiles in HepG2 Cells," American Journal of Chinese Medicine, Vol. 36, No. 4, 2008, pp. 783-797. http://dx.doi.org/10.1142/S0192415X08006235

[54] Z. Sun, Y. H. Su and X. Q. Yue, "Professor Ling Changquan's Experience in Treating Primary Liver Cancer: An Analysis of Herbal Medication," Journal of Chinese Integrative Medicine, Vol. 6, No. 12, 2008, pp. 1221-1225. http://dx.doi.org/10.3736/jcim20081203

[55] Z. Zhang, H. F. Que, Y. Y. Zhu, Y. F. Wang, X. D. Liu and P. Y. Zheng, "Effects of Chinese Herbs for Replenishing Qi and Resolving Stagnation on Transforming Growth Factor-Beta1 of Skin Ulcers in Rats with Diabetes," Journal of Chinese Integrative Medicine, Vol. 5, No. 4, 2007, pp. 416-420. http://dx.doi.org/10.3736/jcim20070411

[56] W. Cao and A. G. Zhao, "Prescription Rules of Chinese Herbal Medicines in Treatment of Gastric Cancer,” Journal of Chinese Integrative Medicine, Vol. 7, No. 1, 2009, pp. 1-8. http://dx.doi.org/10.3736/jcim20090101

[57] Z. Y. Gao, H. Xu, D. Z. Shi, C. Wen and B. Y. Liu, “Anal- ysis on Outcome of 5284 Patients with Coronary Artery Disease: The Role of Integrative Medicine,” Journal of Ethnopharmacology, Vol. 141, No. 2, 2012, pp. 578-583. http://dx.doi.org/10.1016/j.jep.2011.08.071

[58] Y. Xiao, F. Q. Yang, S. P. Li, G. Hu, S. M. Lee and Y. T. Wang, "Essential Oil of Curcuma wenyujin Induces Apoptosis in Human Hepatoma Cells,” World Journal of Gastroenterology, Vol. 14, No. 27, 2008, pp. 4309-4918. http://dx.doi.org/10.3748/wjg.14.4309

[59] Y. L. Huang, “Cupping-Bloodletting Therapy of Saudi Arabia and Its Clinical Application," Chinese Acupuncture \& Moxibustion, Vol. 28, No. 5, 2008, pp. 375-377.

[60] K. Vanderploeg and X. Yi, "Acupuncture in Modern Society," Journal of Acupuncture and Meridian Studies, Vol. 2, No. 1, 2009, pp. 26-33.

[61] L. Y. Chen and S. Y. Yan, "Embodiment of 'Harmony' in the Concept of Well-Being of China," Chinese Journal of Medical History, Vol. 40, No. 5, 2010, pp. 274-277.

[62] M. Krishnaveni and S. Mirunalini, "Therapeutic Potential of Phyllanthus emblica (amla): The Ayurvedic Wonder," Journal of Basic and Clinical Physiology and Pharmacology, Vol. 21, No. 1, 2010, pp. 93-105.

[63] L. Pari and S. Venkateswaran, "Protective Role of Phaseolus vulgaris on Changes in the Fatty Acid Composition in Experimental Diabetes," Journal of Medicinal Food, Vol. 7, No. 2, 2004, pp. 204-209. http://dx.doi.org/10.1089/1096620041224120 